\title{
Completeness and Legibility of Handwritten Prescriptions in Sana'a, Yemen
}

\author{
Yaser Mohammed Al-Worafia, b Rahul P. Patel ${ }^{c}$ Syed Tabish Razi Zaidic \\ Wafa Mohammed Alseragi $^{d}$ Masaad Saeed Almutairi ${ }^{\mathrm{e}}$ Ali Saleh Alkhoshaiban ${ }^{\mathrm{e}}$ \\ Long Chiau Ming ${ }^{c, f}$
}

a Department of Clinical Pharmacy, Faculty of Pharmacy, University of Science and Technology, Sana'a, Yemen;

${ }^{b}$ Department of Clinical Pharmacy, College of Pharmacy and Health Sciences, Ajman University, Ajman,

United Arab Emirates; ' Division of Pharmacy, School of Medicine, University of Tasmania, Hobart, TAS, Australia;

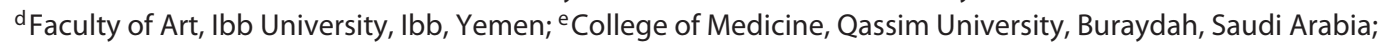

${ }^{f}$ School of Pharmacy, KPJ Healthcare University College, Nilai, Malaysia

\section{Significance of the Study}

- This study showed that the quality of handwritten prescriptions in Sana'a, Yemen, was very poor, and could therefore easily lead to dispensing errors. This highlights the need for introducing computerized physician order entries into general practice. This would improve the dispensing system because only $10 \%$ of the community pharmacies in Yemen have qualified pharmacists.

\section{Keywords}

Prescribing error $\cdot$ Medication safety $\cdot$ Medication error . Computerized physician order entry · Pharmacy practice

\section{Abstract}

Objective: The aim of this study was to investigate the completeness and legibility of prescriptions dispensed in community pharmacies located in Sana'a, Yemen. Materials and Methods: A cross-sectional study was conducted at 23 randomly selected community pharmacies throughout the capital city of Sana'a, Yemen, from May 2015 to January 2016. A total of 2,178 prescriptions were analyzed for the

\section{KARGER}

E-Mail karger@karger.com www.karger.com/mpp essential elements of a complete prescription using a validated checklist. Results: Of the 2,178 prescriptions, 19 $(0.87 \%)$ were considered to be of good quality. The remaining 2,159 (99.12\%) were considered as being of very poor quality. Writing errors relating to patients and prescribed medications were the most common errors. Conclusion: In this study, the quality of prescription writing was found to be very poor. Hence, continuous professional development programs are recommended to improve the quality of pre-

Yaser Mohammed Al-Worafi and Long Chiau Ming contributed equally to this work.
Yaser Mohammed Al-Worafi

Faculty of Pharmacy, University of Science and Technology 60 Meters Road

Sana'a (Yemen)

E-Mailyworafi@yahoo.com 
scription writing among physicians. Future studies in other cities and investigation of the impact of continuous educational programs on the quality of prescription writing are strongly recommended.

(c) 2018 The Author(s)

Published by S. Karger AG, Basel

\section{Introduction}

Good-quality prescriptions are very important to minimize errors in the dispensing of medicines; physicians should adhere to the guidelines for prescription writing in order to provide quality treatment to patients $[1,2]$. All prescriptions should contain accurate and appropriate information about the patient and the medication that is being prescribed. All prescriptions should contain information such as the prescriber's name, address, telephone number, and signature; the patient's name, address, age, and weight; the prescription date; the name, formulation, strength, dose, frequency of administration, quantity of the prescribed drug, and instructions for its use as well as the diagnosis/indications $[3,4]$. The aim of this study was to investigate the completeness and legibility of prescriptions dispensed by community pharmacies in Sana'a, Yemen.

\section{Materials and Methods}

This was a cross-sectional study conducted in the city of Sana'a, Yemen, using the cluster sampling method. A sampling frame was prepared using the list of registered pharmacies located at different areas within Sana'a, and a random number table was used. A total of 23 community pharmacies were selected, taking their geographical location into consideration. A minimum of one pharmacy dispenser or pharmacist from each pharmacy participated in this study. The prescriptions dispensed at the selected pharmacies from May 2015 to January 2016 were analyzed by 2 trained, independent researchers-cum-pharmacists. By using a validated checklist, prescriptions were analyzed for whether the required information was included in the prescriptions [3].

The checklist contained the following prescription errors related to (a) physician: name, contact details, and signature (3 criteria); (b) patient information: name, address, age, gender, and weight (5 criteria); (c) prescribed medications: drug name, strength, dose units, dosage form, quantity of medications, duration of therapy, route of administration, dose interval, instructions, drug abbreviation, unit abbreviation, and spelling mistakes (12 criteria); and (d) prescription: date of prescription, diagnosis, and clarity of prescription/legibility (3 criteria). If both investigators were unable to decipher any of the information included in the prescription, it was considered illegible. Each prescription was scored based on the compliance to the above 23 parameters, with
Table 1. Type and frequency of writing errors found in 2,178 prescriptions

\begin{tabular}{lc}
\hline Type of error & Frequency, $n$ (\%) \\
\hline Physician-related information & \\
Name & $26(1.19)$ \\
Contact details & $39(1.79)$ \\
Signature & $51(2.34)$ \\
Patient-related information & \\
Weight & $2,173(99.77)$ \\
Address & $2,102(96.51)$ \\
Age & $1,524(69.97)$ \\
Gender & $1,391(63.86)$ \\
Name & $482(22.13)$ \\
Prescribed medication-related information & \\
Spelling & $2,124(97.52)$ \\
Instructions for use & $2,067(94.90)$ \\
Quantity & $1,908(87.60)$ \\
Dose interval & $1,824(83.74)$ \\
Strength & $1,645(75.52)$ \\
Route of administration & $1,608(73.82)$ \\
Dose units & $1,573(72.22)$ \\
Duration of therapy & $1,433(65.79)$ \\
Drug name & $1,098(50.41)$ \\
Dosage form & $961(44.12)$ \\
Drug abbreviations & $456(20.93)$ \\
Unit abbreviations & $811(37.23)$ \\
Prescription-related information & \\
Clarity of prescription/legibility & $1,919(88.10)$ \\
Diagnosis/indication & $1,770(81.26)$ \\
Date of prescription & $1,619(74.33)$ \\
\hline & \\
\hline
\end{tabular}

1 point given for each erroneous criterion. The quality of the prescription was scored as: very poor, $\geq 10$ errors; poor, 3-9 errors; fair, 2 errors; good, 1 error [3].

A total of 2,178 prescriptions were analyzed. The data obtained were descriptively analyzed using the Statistical Package for the Social Sciences ${ }^{\circledR}$ (SPSS) v15 (Chicago, IL, USA). The Research Ethics Committee of the College of Pharmacy, University of Science and Technology, Yemen, approved the study. The investigators received permission to conduct the survey from the community pharmacy managers. Written informed consent was obtained from the participants. No personal information about respondents, pharmacists, physicians, or patients was collected and strict confidentiality was observed.

\section{Results}

Of the 2,178 prescriptions, only $19(0.87 \%)$ were considered as being of good quality. The remaining 2,159 (99.12\%) were considered to be of very poor quality; 1,770 (81.26\%) were incomplete without indication or diagnosis and 1,919 (88.10\%) were partly illegible. Most errors 
were related to spelling $(2,124$ or $97.52 \%)$, instructions on drug use $(2,067$ or $94.90 \%)$, and dose intervals $(1,824$ or $83.74 \%)$. Errors concerning information about the patient (e.g., body weight) and the spelling of the name of the prescribed medication were the most common, and the spelling of the name of the prescriber was the least common (Table 1).

\section{Discussion}

In this study, $99.12 \%$ of the screened prescriptions were considered to be of very poor quality. The number of errors ranged from 5 to 20 per prescription. Errors related to patients and prescribed medications were the most common while those related to the physicians were the least common. These findings were similar to those of a previous study conducted in a tertiary care hospital in Yemen, where 1,904 prescriptions were screened and 99.12\% were considered as being of poor quality, but the errors in the contact details of physicians were one of the most common [3].

Preventing prescription writing errors is very important to ensure the safety of patients. For example, a study that involved 24,767 prescriptions revealed that pharmacists spent 8 times longer for intervention and interpretation when dealing with handwritten prescriptions than when prescriptions were prepared using computerized physician order entry. Hence, the use of computerized physician order entry has proven to be effective in eliminating prescribing errors $[5,6]$. It augurs well for the Ye- meni dispensing system because only $10 \%$ of community pharmacies have qualified pharmacists, with the remaining 90\% handled solely by technicians [7-9].

The major limitation of this study was that it was conducted in only one city in Yemen. Future studies investigating the impact of training doctors how to prescribe, and the pilot implementation of computerized physician order entry on the quality of prescription writing in all major cities in Yemen are strongly recommended. Furthermore, prescription writing errors could be prevented by retraining doctors on prescribing and common errors in writing prescriptions. Continuous professional educational programs for doctors, pharmacists, and technicians could improve the quality of prescription writing as well as the proficiency of prescription screening before dispensing medications to patients.

\section{Conclusion}

This study indicated that the quality of handwritten prescription in the city of Sana'a was very poor. Hence, it is important to devise means of improving the quality of prescription writing among physicians.

\section{Acknowledgement}

The authors would like to thank the pharmacy managers and staff members who agreed to participate in the study. Special thanks to pharmacists Waheeb Ali and Ali Alhussaini for their help.

\section{References}

$\checkmark 1$ Meyer TA: Improving the quality of the order-writing process for inpatient orders and outpatient prescriptions. Am J Health Syst Pharm 2000;57(suppl 4):S18-S22.

2 Guide to Good Prescribing: A Practical Manual. Geneva, World Health Organization, 1994.

3 Al-Worafi YM: Prescription writing errors at a tertiary care hospital in Yemen: prevalence, types, causes and recommendations. Am J Pharm Health Res 2014;2:134-140.
4 Davis L, Brunetti L, Lee EK, et al: Effects of computerized physician order entry on medication turnaround time and orders requiring pharmacist intervention. Res Social Adm Pharm 2014;10:756-767.

5 Tang KL, Wimmer BC, Akkawi ME, et al: Incidence and pattern of medication errors in a general paediatric ward in a developing nation. Res Social Adm Pharm 2018;14:317319.
6 Salmasi S, Wimmer BC, Khan TM, et al: A proposed stepwise approach to reducing medication errors in older people. Res Social Adm Pharm 2018;14:207-209.

7 Al-Worafi YM: Pharmacy practice and its challenges in Yemen. Australas Med J 2014;7: $17-23$.

-8 Al-Worafi YM: The challenges of pharmacy education in Yemen. Am J Pharm Educ 2014; 78:146-150.

9 Al-Worafi YM: Pharmacy education in Yemen. Am J Pharm Educ 2013;77:65. 